\title{
Lymphadenopathy Due to Penicillium Marneffei Infection: Diagnosis by Fine Needle Aspiration Cytology
}

Benjaporn Chaiwun, M.D., Surapan Khunamornpong, M.D., Chusak Sirivanichai, M.D., Samreung Rangdaeng, M.D., Khuanchai Supparatpinyo, M.D., Jongolnee Settakorn, M.D., Charin Ya-in, M.D., Paul Thorner, MD, Ph.D. Departments of Pathology (BC, SK, SR, JS, CY), Radiology (CS), and Internal Medicine (KS), Faculty of Medicine, Chiang Mai University, Chiang Mai, Thailand; and Division of Pathology (PT), Hospital for Sick Children and Department of Laboratory Medicine and Pathobiology, University of Toronto, Toronto, Ontario, Canada

Penicillium marneffei is an opportunistic fungal infection that usually causes disseminated disease, mainly in immunocompromised individuals, especially those with HIV infection. Untreated cases are usually fatal. Diagnosis is traditionally made by biopsy and/or culture; successful diagnosis by fine needle aspiration (FNA) has only been reported once. We present eight cases of HIV-infected patients with lymphadenopathy caused by $P$. marneffei infection, in which the diagnosis was made by FNA. In all cases, intracellular and extracellular yeast forms were visualized, and the characteristic cross-septation of $P$. marneffei was highlighted by GMS staining. All diagnoses were confirmed by culture. Anti-fungal treatment for $\boldsymbol{P}$. marneffei was initiated, resulting in marked clinical improvement. We conclude that a diagnosis of lymphadenopathy caused by $P$. marneffei can reliably be made by FNA. The diagnosis is more rapid than biopsy or culture, allowing rapid institution of therapy, particularly important in immunocompromised patients. In all our cases, not only were lymphoma and other causes of lymphadenopathy ruled out, but also the necessity for an open surgical biopsy was obviated. This can be especially beneficial to patients (e.g., three in our study) in which lymphadenopathy is confined to deep intra-abdominal nodes.

KEY WORDS: Fine needle aspiration, HIV, Lymphadenopathy, Penicillium marneffei.

Mod Pathol 2002;15(9):939-943

Copyright $(\odot 2002$ by The United States and Canadian Academy of Pathology, Inc.

VOL. 15, NO. 9, P. 939, 2002 Printed in the U.S.A.

Date of acceptance: May 22, 2002.

Address reprint requests to: Benjaporn Chaiwun, M.D., Department of

Pathology, Faculty of Medicine, Chiang Mai University, Chiang Mai 50200,

Thailand; e-mail: bchaiwun@mail.med.cmu.ac.th; fax: 66-53-217144.

DOI: 10.1097/01.MP.0000027203.44333.95
Penicillium marneffei infection is endemic in Southeast Asia and Southern China (1-3). It was first described in 1959 (4) and the first natural infection in humans was reported in 1973 in an American who had visited Southeast Asia (5). By 1988, there was a total of only 21 reported cases (2). Also at this time, the first case associated with HIV was reported (6) and since then, penicilliosis has emerged as one of the most common infections in HIV-infected patients residing in endemic areas (3, 7-12). Most cases of penicilliosis are disseminated in nature (2), with fever, generalized lymphadenopathy, hepatosplenomegaly, mucosal ulcers and skin lesions (7-10, 13-15). The infection responds to anti-fungal treatment, but untreated cases, especially in immunocompromised patients, are usually fatal (10); hence, rapid diagnosis is of great benefit to these patients.

Lymphadenopathy is a common diagnostic problem in immunocompromised patients, and can result from a variety of causes, including one or more infectious agents and malignancy, especially lymphoma. Fine needle aspiration (FNA) is often used to investigate this problem since it offers several advantages over biopsy, including a more rapid diagnosis, less morbidity, and greater ease of accessibility to deep lymph nodes. Despite the advantages of FNA, successful use of this technique to diagnose Penicillium marneffei infection has only been reported once (16). To illustrate the diagnostic criteria, reliability and advantages of this approach, we report eight cases in which $P$. marneffei infection was diagnosed by fine needle aspiration of lymph nodes. FNA results led to prompt institution of anti-fungal treatment and, in one patient, additional testing that uncovered a previously unsuspected HIV infection.

\section{MATERIALS AND METHODS}

Eight cases of lymphadenopathy due to $P$. marneffei were collected from the archives of the 
Department of Pathology, Faculty of Medicine, Chiang Mai University, Chiang Mai, Thailand from 1996-2001. During this period, a total of 56 aspirates were received from HIV-positive patients. No cases of penicilliosis were received from HIVnegative patients. No complications were observed as a result of performing these procedures. In all eight cases, diagnoses were based solely upon cytological material obtained by FNA. Specimens were obtained by a radiologist under imaging guidance for deep-seated lymph nodes and by direct palpation for superficial lymph nodes. Papanicolaou, Diff-Quik, GMS, mucicarmine and ZiehlNeelsen stains were performed on all cases. All eight cases were submitted as well for fungal culture for verification. No case was found in which penicilliosis was diagnosed by culture and missed on fine needle aspirate. The data on the eight patients are presented in Table 1 . Cases 1 and 2 are presented in greater detail since each case illustrates a point with respect to clinical management.

\section{Case 1}

A 4-year-old girl, who was diagnosed to have HIV infection at two years of age, presented with chronic diarrhea. Two weeks before the admission, she had developed generalized skin papules and lymphadenopathy. In addition to these findings, physical examination also revealed splenomegaly. Laboratory studies showed a hemoglobin concentration of $7.8 \mathrm{~g} / \mathrm{dl}$, leukocyte count of $2350 / \mathrm{mm}^{3}$, with a differential count of $15 \%$ neutrophils and $85 \%$ lymphocytes, and platelet count of $185,000 / \mathrm{mm}^{3}$. Ultrasonography of the abdomen demonstrated diffuse splenomegaly and enlarged para-aortic and paravertebral lymph nodes. A diagnosis of $P$. marneffei infection was established on material obtained from scraping one of the skin lesions. FNA of para-aortic nodes under imaging guidance was performed to verify the cause of lymphadenopathy, in particular to rule out a lymphoma and other infectious causes. The aspirate confirmed a diagnosis of $P$. marneffei infection. Her clinical symptoms ameliorated following antifungal treatment.

\section{Case 2}

A 38-year-old woman presented with abdominal pain for 4 days. She had been losing weight for the past few months. Physical examination revealed a 5 $\mathrm{cm}$ ill-defined firm mass with mild tenderness in the left upper quadrant of the abdomen. Laboratory findings included a hemoglobin concentration of $10 \mathrm{~g} / \mathrm{dL}$, leukocyte count of $5400 / \mathrm{mm}^{3}$, with differential count of $74 \%$ neutrophils, $18 \%$ lymphocytes, $6 \%$ monocytes and $2 \%$ eosinophils, and platelet count of $316,000 /$ $\mathrm{mm}^{3}$. Ultrasonography of the abdomen revealed mesenteric lymphadenopathy. FNA of an intraabdominal lymph node was performed under imaging guidance. The aspirate revealed the presence of $P$. marneffei. Anti-fungal therapy for penicilliosis was commenced, resulting in clinical improvement. Because of the diagnosis of penicilliosis, the patient was subsequently tested for HIV and found to be positive by ELISA testing of her serum.

\section{RESULTS}

\section{Cytologic Findings}

Three FNA specimens were obtained from deep seated lymph nodes (para-aortic and mesenteric) under ultrasound guidance (Fig. 1). The remaining five specimens were obtained from superficial lymph nodes (cervical, supraclavicular and inguinal) under direct palpation. The cytologic materials from all eight patients revealed similar findings. By Papanicolaou staining, mixed inflammatory cells, including small lymphocytes, plasma cells and histiocytes were observed within a necrotic background. Some histiocytes were packed with intracytoplasmic small oval-shaped organisms. Various numbers of $P$. marneffei were scattered in the background.(Fig. 2A-B). By Papanicolaou and Diff-Quik staining, a central clear transverse septum could be identified (Fig. 3, A and B, respectively). The organ-

TABLE 1. Clinical Data of Patients with Penicilliosis by FNA

\begin{tabular}{|c|c|c|c|c|c|}
\hline Case & Age & Sex & Clinical Presentation & HIV status & FNA site \\
\hline 1 & $4 \mathrm{yr}$ & $\mathrm{F}$ & $\begin{array}{l}\text { Chronic diarrhea, skin papules, para- } \\
\text { aortic lymphadenopathy }\end{array}$ & Positive $\times 2 \mathrm{yr}$ & Para-aortic LN \\
\hline 2 & $38 \mathrm{yr}$ & $\mathrm{F}$ & $\begin{array}{l}\text { Abdominal pain } \times 4 \text { days, mesenteric } \\
\text { lymphadenopathy }\end{array}$ & Positive (tested post-FNA) & Mesenteric LN \\
\hline 3 & $31 \mathrm{yr}$ & $\mathrm{F}$ & $\begin{array}{l}\text { Abdominal pain } \times 2 \text { wks, para-aortic } \\
\text { and mesenteric lymphadenopathy }\end{array}$ & Positive $\times 5 \mathrm{yr}$ & Para-aortic LN \\
\hline 4 & $37 \mathrm{yr}$ & M & Fever, supraclavicular lymphadenopathy & Positive $\times 2 \mathrm{yr}$ & Supraclavicular LN \\
\hline 5 & $29 \mathrm{yr}$ & $\mathrm{F}$ & $\begin{array}{l}\text { Chronic tonsillitis and sinusitis, cervical } \\
\text { lymphadenopathy }\end{array}$ & Positive $\times 6 \mathrm{yr}$ & Cervical LN \\
\hline 6 & $31 \mathrm{yr}$ & $\mathrm{F}$ & Fever, cervical lymphadenopathy & Positive $\times 2 \mathrm{yr}$ & Cervical LN \\
\hline 7 & $29 \mathrm{yr}$ & $\mathrm{F}$ & Fever, cervical lymphadenopathy & Positive $\times 1 \mathrm{yr}$ & Cervical LN \\
\hline 8 & $28 \mathrm{yr}$ & M & Fever, inguinal lymphadenopathy & Positive $\times 1 \mathrm{yr}$ & Inguinal LN \\
\hline
\end{tabular}




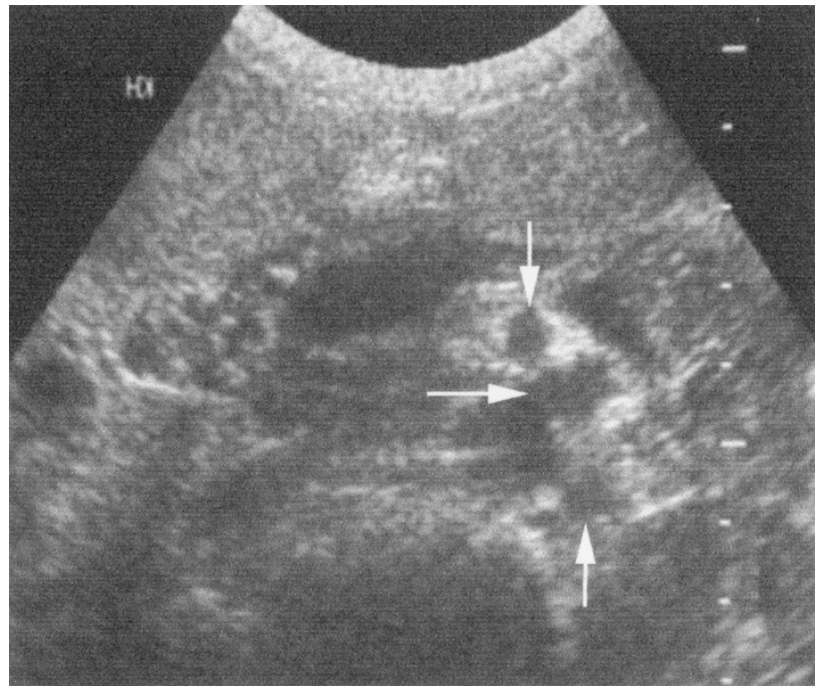

FIGURE 1. Ultrasonogram of the abdomen of patient 3 revealing multiple enlarged para-aortic lymph nodes appearing as echo-lucent zones (arrows).

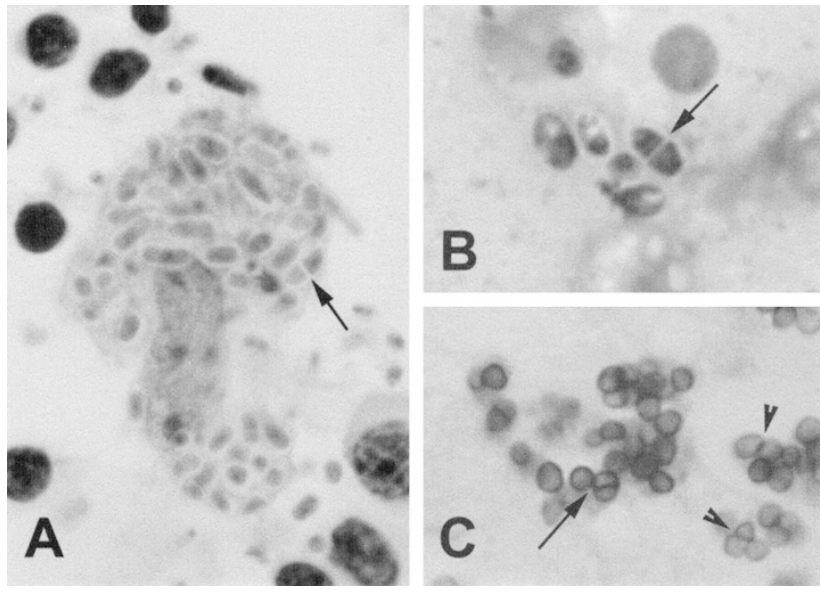

FIGURE 3. Higher magnification showing the details of $P$. marneffei. The characteristic intracytoplasmic transverse septum (arrow) appears clear on Papanicolaou (A) and Diff-Quik staining (B) can be highlighted by GMS staining (C). Division by fission can also be seen (arrowhead) $($ all $\times 1000)$.

\section{DISCUSSION}

P. marneffei is an endemic organism in Southeast Asia and Southern China. It is a dimorphic fungus exhibiting a mycelial form at $25^{\circ} \mathrm{C}$ and yeast form at $37^{\circ} \mathrm{C}(4,14)$. The mycelial form produces a diffusable red pigment in culture (17). The yeast cells reproduce by fission rather than by budding, which serves as an aid for diagnosis in biopsy and FNA material. The fungus can cause infection in either normal or immunocompromised hosts $(9,17)$. Most cases of penicilliosis are disseminated infections (2) clinically resembling histoplasmosis and leishmaniasis $(15,18)$. The common clinical features include fever, weight loss, anemia, generalized lymphadenopathy, hepatosplenomegaly, pulmonary involvement, mucosal ulcers and skin lesions $(7-10,13-15)$. Occasionally there is bone and joint involvement $(19,20)$. The skin lesions are usually molluscum-like papules with necrosis $(10,18)$. Although the fungus can infect apparently healthy individuals (2), it is most commonly seen nowadays in immunocompromised patients, especially in HIV-infected individuals residing in endemic areas (7-12). Studies from these regions report up to $93 \%$ of patients with penicilliosis also have $\operatorname{HIV}(7,10)$.

The manner in which humans acquire P. marnef$f e i$ infection is unclear. Animal reservoirs in northern Thailand have been identified to include the bamboo rat (Rhizomys sinensis), the hoary bamboo rat (Rhizomys pruinosus), the large bamboo rat (Rhizomys sumatrensis), and the small bay bamboo rat (Cannomys badius) (21-24). The organism can be isolated from lungs, spleen, liver and lymph nodes in more than $90 \%$ of Rhizomys species $(2,21$, $23,24)$. While exposure to soil has been suggested as a risk factor (25), another study found that $P$. 
marneffei is not present in soil except around animal burrows (24). It remains unclear whether rats are involved in transmitting the organism to humans, or whether both humans and rats are infected from a common but still unidentified source (2).

Infected tissues can show a mixture of reactions, including necrosis, suppuration and granuloma formation (2). Granulomata are often poorly formed or may exist only as a diffuse infiltrate of macrophages $(9,17,26)$. Necrosis with lymphocyte depletion is more commonly seen in immunocompromised patients (2). In infected organs, $P$. marneffei can be found in both intracellular and extracellular locations (10). Lymph nodes may contain a great number of organisms, mainly within histiocytes (17). The yeast-like organisms can be spherical, oval or sausage-shaped (17). Their size varies from 3-8 microns in length $(10,17)$. Within cells, they tend to be more uniform at 3 microns, whereas outside the histiocytes, the fungi elongate up to 8 microns (17). A characteristic feature is an intracytoplasmic transverse septum that is clear on routine stains such as Diff-Quik and Papanicolaou $(10,22)$ but can be highlighted by GMS and periodic acid-Schiff stains $(17,27)$.

Given the presence of small yeast-like organisms in either Papanicolaou or Diff-Quik stained material, other organisms need to be ruled out, including Histoplasma, Cryptococcus, and Cañdida. Histoplasma is the organism most commonly confused with $P$. marneffei, but can be distinguished by three criteria. First, the intracytoplasmic transverse septum, best demonstrated by GMS stain, is characteristic for P. marneffei. Second, the finding of budding yeast is incompatible with a diagnosis of $P$. marneffei which reproduce by fission, in contrast to several other fungi (19). Finally, oval and sausage forms are not seen with Histoplasma (17). Another possible source of error is that by GMS staining, the yeast forms of $P$. marneffei can have a central or eccentric dot and can be confused with Pneumocystis carinii. The latter can be distinguished from $P$. marneffei by a mainly extracellular location, the presence of crescent forms, and the lack of septation (8).

The diagnosis is usually made by culture of infected material or by histologic examination of a skin biopsy, lymph node biopsy, or bone marrow aspirate/biopsy (10). There are reports of penicilliosis diagnosed by needle biopsy of lung (28) and liver $(29,30)$. Diagnoses based on culture or histologic sections take several days before a diagnosis can be rendered. Diagnoses based on cytologic material are much more rapid and, for P. marneffei, usually involve touch preparations from skin scrapings (7). There is one report of a diagnosis made on fluid obtained by bronchoalveolar lavage (31). Sur- prisingly, there is only one reported cases of a successful FNA diagnosis of $P$. marneffei infection, in this case from a lymph node of an HIV-positive Chinese patient (16). One additional case was diagnosed retrospectively in an FNA specimen after a subsequent lymph node biopsy showed the presence of the organism (32). The lack of reports is not a reflection of the rarity of the disease, at least in endemic areas. On the contrary, about one third of lymph node biopsies from HIV-positive patients in our hospital were found to have penicilliosis on biopsy (26). Considering FNA specimens on lymph nodes alone, $\sim 14 \%$ of cases from HIV-positive patients were positive for penicilliosis in our institution. The lower percentage with aspirate specimens may reflect the fact that there is more tissue available in a biopsy yielding a higher number of cases of penicilliosis.

Our eight cases illustrate many of the advantages of using FNA, especially in the management of HIVinfected patients. In experienced hands, FNA is reliable and accurate for making a diagnosis of $P$. marneffei infection. In our patients, $P$. marneffei infection was rapidly diagnosed from FNA of lymph nodes. Rapid diagnosis by FNA cytology is of great benefit to HIV-positive patients, since many infections have specific treatments that can be started promptly, eliminating the need for surgical biopsy. In our cases, anti-fungal therapy was instituted immediately after the diagnosis was made. This is clinically important since treated patients with $P$. marneffei infection usually recover, whereas untreated patients usually die (10). All our patients recovered from their infection.

While diagnosis of $P$. marneffei infection can be rapidly made on scrapings from skin lesions, these are not present in about one third of cases (3). Moreover, in HIV-infected patients, lymphadenopathy is very common and could result from a variety of causes including the persistent generalized lymphadenopathy of HIV-infection, malignancies such as lymphoma, and single or multiple infections, the most common of which in Southeast Asia are tuberculosis, penicilliosis and cryptococcosis $(12,26)$. Hence, even if skin lesions are present, examination of these alone is not sufficient in the work-up of HIV-positive patients and direct lymph node sampling is indicated. This was the situation for case 1 , in which the diagnosis of $P$. marneffei infection was also made on skin scrapings, but other causes of lymphadenopathy still needed to be ruled out. FNA of lymph nodes instead of open biopsy is much more straightforward and less morbid to the patient. This is particularly true for deepseated lymphadenopathy, since access to such lymph nodes by a surgical approach is not a simple outpatient procedure, in contrast to FNA. While FNA material may be suboptimal for diagnosis of 
lymphoma, the infectious causes of lymphadenopathy are more readily diagnosed, and the remaining patients for whom the FNA results are indeterminate can proceed to open biopsy.

Final, the currently frequent association of HIV and $P$. marneffei infections suggests some guidelines for patient management. Patients with $P$. marneffei infection should be suspected of also being infected with HIV. Case 2 exemplifies this point; the diagnosis of HIV was not made (or considered) until after $P$. marneffei was identified by FNA. The corollary is also true, that HIV patients are at risk for $P$. marneffei infection $(8,9)$. This risk is not restricted to those living in endemic areas. HIVinfected individuals who travel to Southeast Asia and Southern China can also become infected by $P$. marneffei $(15,27,33)$. Since the number of HIVinfected patients are increasing around the world, all countries need to be aware of $P$. marneffe $i$ as a potential pathogen in their immunocompromised patient population.

\section{REFERENCES}

1. Deng Z, Yun M, Ajello L. Human penicilliosis marneffei and its relation to the bamboo rat (Rhizomys pruinosus). J Med Vet Mycol 1986;24:383-9.

2. Deng Z, Ribas J, Gibson D, Connor D. Infections caused by Penicillium marneffei in China and Southeast Asia: review of eighteen published cases and report of four more Chinese cases. Rev Infect Dis 1988;10:640-52.

3. Cooper CJ, McGinnis M. Pathology of Penicillium marneffei. An emerging acquired immunodeficiency syndrome-related pathogen. Arch Pathol Lab Med 1997;121:798-804.

4. Segretain G. Description d'une nouvelle espèce de penicillium. Penicillium marneffei n, sp. Bull Soc Mycol Fr 1959;75: 412-6.

5. DiSalvo A, Fickling A, Ajello L. Infection caused by Penicillium marneffei: description of first natural infection in man. Am J Clin Pathol 1973;60:259-63.

6. Piehl M, Kaplan R, Haber M. Disseminated penicilliosis in a patient with acquired immunodeficiency syndrome. Arch Pathol Lab Med 1988;112:1262-4.

7. Supparatpinyo K, Chiewchanvit S, Hirunsri P, Uthammachai C, Nelson K, Sirisanthana T. Penicillium marneffei infection in patients infected with human immunodeficiency virus. Clin Infect Dis 1992;14:871-4.

8. Tsui W, Ma K, Tsang D. Disseminated Penicillium marneffei infection in HIV-infected subject. Histopathology 1992;20: 287-93.

9. Hilmarsdottir I, Meynard J, Rogeaux O, Guermonprez G, Datry A, Katlama C, et al. Disseminated Penicillium marneffei infection associated with human immunodeficiency virus: a report of two cases and a review of 35 published cases. J Acquir Immune Defic Syndr 1993;6:466-71.

10. Supparatpinyo K, Khamwan C, Baosoung V, Nelson K, Sirisanthana T. Disseminated Penicillium marneffei infection in southeast Asia. Lancet 1994;344:110-3.

11. Duong T. Infection due to Penicillium marneffei, an emerging pathogen: review of 155 reported cases. Clin Infect Dis 1996;23:125-30.

12. Ruxrungtham K, Phanuphak P. Update on HIV/AIDS in Thailand. J Med Assoc Thai 2001;84 Suppl 1:S1-17.

13. Imwidthaya P. Update of Penicillosis marneffei in Thailand. Mycopathologia 1994;127:135-7.
14. Sekhon A, Stein L, Garg A, Black W, Glezos J, Wong C. Pulmonary Penicillosis marneffei: report of the first imported case in Canada. Mycopathologia 1994;128:3-7.

15. Kok I, Veenstra J, Rietra P, Dirks-Go S, Blaauwgeers J, Weigel H. Disseminated Penicillium marneffei infection as an imported disease in HIV-1 infected patients. Description of two cases and a review of the literature. Neth J Med 1994;44:18-22.

16. Ma K, Tsui M, Tsang D. Fine needle aspiration diagnosis of Penicillium marneffei infection. Acta Cytol 1991;35:557-9.

17. Deng Z, Connor D. Progressive disseminated penicilliosis caused by Penicillium marneffei. Report of eight cases and differentiation of the causative organism from Histoplasma capsulatum. Am J Clin Pathol 1985;84:323-7.

18. Imwidthaya P. Systemic fungal infections in Thailand. J Med Vet Mycol 1994;32:395-9.

19. Jayanetra P, Nitiyanant P, Ajello L, Padhye A, Lolekha S, Atichartakarn V, et al. Penicilliosis marneffei in Thailand: report of five human cases. Am J Trop Med Hyg 1984;33:637-44.

20. Louthrenoo W, Thamprasert K, Sirisanthana T. Osteoarticular penicilliosis marneffei. A report of eight cases and review of the literature. Br J Rheumatol 1994;33:1145-50.

21. Li J, Pan L, Wu S. Mycologic investigation on Rhizomys pruinous senex in Guangxi as natural carrier with Penicillium marneffei. Chin Med J 1989;102:477-85.

22. Pracharktam R, Sriurairatna S, Jayanetra P. Morphological variation in pathogenic strains of Penicillium marneffei. J Med Assoc Thai 1992;75 Suppl 1:172-9.

23. Ajello L, Padhye A, Sukroongreung S, Nilakul C, Tantimavanic S. Occurrence of Penicillium marneffei infections among wild bamboo rats in Thailand. Mycopathologia 1995;131:1-8.

24. Chariyalertsak S, Vanittanakom P, Nelson K, Sirisanthana T, Vanittanakom N. Rhizomys sumatrensis and Cannomys badius, new natural animal hosts of Penicillium marneffei. J Med Vet Mycol 1996;34:105-10.

25. Chariyalertsak S, Sirisanthana T, Supparatpinyo K, Praparattanapan J, Nelson K. Case-control study of risk factors for Penicillium marneffei infection in human immunodeficiency virus-infected patients in northern Thailand. Clin Infect Dis 1997;24:1080-6.

26. Bhoopat L, Thamprasert K, Chaiwun B, Attasiri C, Vithayasai $\mathrm{P}$, Chaimongkol B, et al. Histopathologic spectrum of AIDSassociated lesions in Maharaj Nakorn Chiang Mai Hospital. Asian Pac J Allergy Immunol 1994;12:95-104.

27. Viviani M, Tortorano A, Rizzardini G, Quirino T, Kaufman L, Padhye A, et al. Treatment and serological studies of an Italian case of Penicilliosis marneffei contracted in Thailand by a drug addict infected with the human immunodeficiency virus. Eur J Epidemiol 1993;9:79-85.

28. Hsu W, Chiang C, Chen C, Hsu J, Chang M. Ultrasoundguided fine needle aspiration biopsy in the diagnosis of chronic pulmonary infection. Respiration 1997;64:319-25.

29. Piratvisuth T, Siripaitoon P, Sriplug H, Ovartlarnporn B. Findings and benefit of liver biopsies in 46 patients infected with human immunodeficiency virus. J Gastroenterol Hepatol 1999;14:146-9.

30. Viriyavejakul P, Rojanasunan P, Viriyavejakul A, Punyarit P, Punpoowong B, Khachansaksumet V, et al. Opportunistic infections in the liver of HIV-infected patients in Thailand: a necropsy study. Southeast Asian J Trop Med Public Health 2000;31:663-7.

31. Chan J, Tsang D, Wong D. Penicillium marneffei in bronchoalveolar lavage fluid. Acta Cytol 1989;33:523-36.

32. Jayaram G, Chew M. Fine needle aspiration cytology of lymph nodes in HIV-infected individuals. Acta Cytol 2000; 44:960-6.

33. Julander I, Petrini B. Penicillium marneffei infection in a Swedish HIV-infected immunodeficient narcotic addict. Scand J Infect Dis 1997;29:320-2. 